\title{
The Effects of Environment on Memory and Reasoning Skills: Comparing Natural and Artificial Environments
}

\author{
Stephen B. Prentice ${ }^{1}$ and Tina M. Waliczek ${ }^{1}$
}

ADDITIONAL INDEX WORDS. attention fatigue, beneficial effects of nature, cognitive function, directed attention, involuntary attention, restoration, restorativeness, voluntary attention

SumMARY. The attention restoration theory suggests that directed attention is subject to fatigue, and the presence of nature and natural environments allows recovery from that fatigue, consequently improving cognitive function. The purpose of this study was to investigate whether the mental processes of memory and reasoning were enhanced when exercised concurrently in a natural environment outdoors vs. an artificial environment such as an indoor classroom or lecture hall. Three hundred and eighty degree-seeking students at Texas State University were tested using modified forms of the Sentence Repetition Test and the California Verbal Learning Test to test verbal memory and a modified form of the Wechsler Adult Intelligence Scale-IV Matrix Reasoning Test to evaluate nonverbal reasoning/fluid intelligence. Half of the subjects (190) were tested in their classroom at the regularly scheduled class time or one located in the same building at a predetermined date and time. Half of the subjects (190) were tested in an outdoor garden classroom at a predetermined date and time. No significant difference was found to exist between in either the memory or reasoning scores of the two groups. However, comparisons of subjects in the same demographic categories produced one significant difference. Students classified as seniors $(P=$ 0.035) who were tested in the natural environment performed significantly better on the Sentence Repetition Test compared with those tested in the artificial environment. Research generally supports the premise that exposure to nature or natural environments can have beneficial effects on physical and mental health and also improve cognitive function. Further studies should possibly include more than one meeting time and additional testing time for participants to sit and observe in the natural vs. artificial environment before testing.

$\mathrm{W}$ illiam James first posited the concept of voluntary attention in 1892 (James, 1892). James suggested this type of attention was consciously engaged when something did not intrinsically attract attention yet was important to address, and one in which effort was expended to perform. For James, voluntary attention not only meant focused concentration but also the suppression of

Received for publication 22 Apr. 2021. Accepted for publication 17 June 2021.

Published online 3 November 2021.

${ }^{1}$ Department of Agricultural Sciences, Texas State University, 601 University Drive, San Marcos, TX 78666

S.B.P. is a Graduate Student.

T.M.W. is a Professor

T.M.W. is the corresponding author. E-mail: tcl0@txstate.edu.

This is an open access article distributed under the CC BY-NC-ND license (https://creativecommons. org/licenses/by-nc-nd/4.0/)

https://doi.org/10.21273/HORTTECH04883-21 potential distractions. Fredrick Law Olmsted, a contemporary of James and considered the father of landscape architecture in the United States, believed that the effort used to sustain voluntary attention might be subject to fatigue and incorporated nature into his urban designs as a remedy for recovery (Kaplan, 1995). Clinical neurologists, recognizing a similar process based on work with patients who had suffered brain damage, have defined the process as directed attention, a term later adopted by Kaplan to avoid confusion with James's terminology (Kaplan, 1995).

Expanding on the observations of James and others, Kaplan and Kaplan (1989) introduced the attention restoration theory. The theory suggested that directed attention is subject to fatigue but can recover by removing attention-demanding activities and resituating oneself within an environment that required only involuntary attention. Involuntary attention is that which requires no effort because that which draws attention does so innately. Again, to avoid confusion with James's terminology, Kaplan (1995) substituted the term fascination for involuntary attention and distinguished two types of fascinations along a hard-soft continuum. A hard fascination is one that draws attention in a dramatic fashion, such as a sudden loud noise or one consistent with imminent danger. A soft fascination is one that draws attention in an undramatic way, such as those experienced while among nature or natural environments. Kaplan (1995) suggested that it is these soft fascinations that provide the best environment for reflection and can most effectively enhance recovery from directed-attention fatigue.

Although fascination is the central element to directed-attention fatigue recovery, Kaplan (1995) described three others: being away, extent, and compatibility. Being away involves removing oneself from habitual activities that require directed attention. The shift can involve a physical escape to a new environment or a conceptual escape as one's gaze or focus looks at a familiar environment in a new way. Extent means that the scope of a restorative environment must present an entirely different perspective and one that is expansive enough to engage as many faculties of thought as possible. Compatibility means that the environment must be consistent with the goals and purposes of the individual. The setting must accommodate the desired outcome, allowing the individual to think and behave naturally without cognitive effort or struggle (Kaplan, 1995).

Kaplan (1995) cited four studies that were particularly notable to the development of the theory. Hartig et al. (1991) compared the pretest and posttest results of a proofreading exercise (which requires a high degree of directed attention) among individuals who had taken a wilderness vacation (backpacking), a nonwilderness vacation (car tours, visiting friends, sightseeing tours), and those who took no vacation and continued to perform everyday activities. The wilderness group showed significant improvement in performance, whereas the nonwilderness and the control groups' performances declined. In a second study, Hartig et al. (1991) reported 
similar results between participants who had been randomly assigned to take a walk in a nature setting or in an urban setting or who sat quietly and read for $40 \mathrm{~min}$, each after being subjected to attention-demanding tasks to induce cognitive fatigue. Not only did members of the nature-walk group perform significantly better on proofreading performance, but they also self-reported a higher degree of perceived restorativeness than did the other two groups.

Cimprich (1993) observed that patients recovering from breast cancer exhibited a high degree of directedattention fatigue. The experimental group participated in 20 -min restorative therapies at least four times per week. The control group participated in no restorative therapy. The experimental group showed significant improvement in performance over the course of the study, whereas the control group's performance declined over the study period.

Lastly, Tennessen and Cimprich (1995) studied how different views from college dormitory windows might influence the attention-restorative process among students. Four categories of views were identified, ranging from all natural to all built. In addition to performing better on a battery of attentional measures, students with a greater natural view from their windows rated themselves better able to perform directed-attention activities than those with built views.

In other studies involving college students, subjects were asked to assess their perceived restorativeness of different environments. Students rated natural settings, scenes of natural settings and urban settings with green space higher in perceived restorativeness when compared with urban settings, built environments, urban settings with no green space, and sports and entertainment settings (Hartig et al., 1997; Herzog et al., 1997; Laumann et al., 2001; Purcell et al., 2001). Felsten (2009) used four categories of scenes to evaluate college students' perceived restorativeness of indoor campus settings used for the purpose of a study break. Actual campus settings were reimaged on a computer screen depicting four categories as background views, as well as classrooms and work areas. The campus scene with no view of nature was ranked the least restorative, whereas the window views of nature, views of land murals, and views of murals with water were measured as the most restorative, respectively.

Two experiments by Berman et al. (2008) evaluated the cognitive benefits of interacting with nature. Results from both experiments found that those who participated in a nature walk or viewed scenes of nature showed a significant improvement in cognitive abilities compared with those who walked in or viewed scenes of urban environments. Additionally, only the nature groups showed significant improvement in their attention levels. Using a similar protocol, Shin et al. (2011) found that subjects who walked in the forest showed significant improvement in cognitive function tests, whereas those who walked in a downtown urban environment did not. Additionally, participants who had walked in the forest exhibited a positive change in mood, whereas those who had walked downtown experienced a negative change.

Using three simulated conditions-a nature walk, a controlled condition, and a perturbation/distress condition-Crossan and Salmoni (2021) also evaluated the cognitive benefits of interacting with nature. Results indicated that only the walk in the simulated natural environment restored directed attention and improved cognitive function. Moreno et al. (2018) reported similar results using nature videos as part of a $\mathrm{K}-2$ school curriculum. Students viewing nature videos significantly improved their academic scores compared with students who did not view the videos.

Research on nature's effect on cognitive function typically incorporates a period of time in which the subject is exposed to a restorative environment to induce recovery from directed-attention fatigue or in which the participants had been exposed to restorative environments for certain periods of time. However, several studies support a coterminous effect of nature on cognitive function. In a study using six landscape conditions with varying degrees of greenness, Jiang et al. (202l) investigated the effects of these landscapes on drivers' mental status and response times while concurrently driving at high speeds. The greener landscapes not only improved cognitive function, but as the landscape's degree of greenness increased, mental status and response times improved, respectively. Adamson and Thatcher (2018) reported that the presence of plants or pictures of plants in offices improved participants' completion time and reduced errors on reading and card-sorting tasks. Kaplan (1993) also suggested that the opportunity to redirect one's focus and experience nature, even for brief moments, can help recovery from directed-attention fatigue.

The purpose of this study was to investigate whether the mental processes of memory and reasoning were enhanced when exercised concurrently in a natural environment and in the presence of soft fascinations (Kaplan, 1995) vs. an artificial environment such as an indoor classroom or lecture hall.

\section{Materials and methods}

SAMPLE. Participants in this study were undergraduate and postgraduate students at Texas State University. Students were either enrolled in a class taught by an instructor who allowed them to voluntarily participate in the study during the class period or those who volunteered to participate to receive extra credit from their instructor at another time and location. On the basis of Krejcie and Morgan's (1970) recommendations for sample size, the targeted sample was 380 participants. Participants were tested in their classroom at the regularly scheduled class time, one located in the same building at a predetermined date and time, or in the outdoor garden classroom at a predetermined date and time and at various times of the semester. Class times and testing times of day differed based on the instructor and classes, but all occurred between 10:00 AM and 5:00 PM. Testing occurred throughout the semester based on when instructors would allow access to their class and students. Although this was not ideal, it was necessary to maximize participation and sample size for the study. Students were allowed to participate only once. Because the sample was not randomly assigned, the results are not necessarily suitable for generalizations beyond this study.

InSTRUMENT. Modified forms of the Sentence Repetition Test (SRT) 
of the Neurosensory Center Comprehensive Examination of Aphasia (Spreen and Benton, 1977) and the California Verbal Learning Testsecond edition (CVLT-II; Delis, 2000) were used to test verbal memory. Verbal memory is the ability to recall spoken information or lists (Bleeker et al., 1988). A modified form of the Wechsler Adult Intelligence Scale-IV (WAIS-IV) Matrix Reasoning Test (Wechsler, 2014) was used to test nonverbal reasoning and fluid intelligence. Nonverbal reasoning is the ability to find patterns and relationships to solve problems (Conway et al., 2003), and fluid intelligence is defined as reasoning and problem-solving abilities independent of previous knowledge (Jaeggi et al., 2008). Modifications to each instrument were necessary to address limitations of time and the manner in which responses were recorded. A panel of experts with expertise in horticulture, social science, and psychology reviewed the modified instrument to ensure validity. All three tests are normally administered in a one-on-one setting, incorporating several trials, and examinees' answers are verbalized. For this study, the testing instruments were modified to allow group administration to be completed within $30 \mathrm{~min}$, and responses were recorded in written form.

EXERCISE 1. The SRT (Spreen and Benton, 1977) involves immediate working memory for sentences. The test is comprised of two lists of 22 sentences of increasing length. The first is a simple one-word sentence such as "Look," and the last, "His interest in the problem increased each time that he looked at the report which lay on the table." The traditional method to administer the test is to read each sentence aloud, and then ask the participant to repeat it out loud to the best of their ability. In the modified form, every other odd-numbered sentence from Form A $(1,5,9$, $13,17,21)$ and Form B $(3,7,11,15$, 19) were read aloud for a total of 11 sentences. After each sentence was read, participants were asked to recall and write the sentence to the best of their ability. The next sentence was read when most of the participants had stopped writing the previous sentence. Ten minutes was allotted for completion of the exercise. A score of one was given for each word correctly recalled in the sequence it was read. Correctness was not contingent upon proper spelling, and there was no penalty for writing words not read. The highest score possible was 107 and the lowest 0 .

Exercise 2. The CVLT-II (Delis, 2000) is a neuropsychological test of episodic verbal learning and memory. The complete CVLT-II comprises several trials involving immediate recall and delayed recall, and free recall and cued recall, of a list of words read to the examinee. The traditional method to administer the CVLT-II is to read aloud sets of 16 words and then ask the participant to repeat out loud as many as possible. In the modified form, only immediate free recall was tested. The 16 words from List A were read aloud across a total of three trials. After each reading, participants were asked to recall the words by writing them to the best of their ability. The next trial began when most of the participants had stopped writing words of the previous trial. Ten minutes was allotted for completion of the exercise. A score of l was given for each word correctly recalled for each trial. Correctness was not contingent on proper spelling. The highest score possible was 48 and the lowest 0 . The terminal score was an aggregate of the three trials.

EXERCISE 3. The WAIS-IV Matrix Reasoning Test (Wechsler, 2014) is used to assess inductive reasoning and fluid intelligence in a nonverbal, visual modality. The test presents a series of matrices from which the participant must determine a rule or pattern relating to the elements that guides the selection of the next appropriate matrix. The problems become more difficult as the test progresses. In a standard testing protocol, 26 series of matrices are presented, and the answer is given verbally. In the modified form, 12 of the series were used. Participants were instructed to choose the correct geometrical design to complete the matrix presented on each page from five possible answers and to record their choice on an answer sheet. Ten minutes was allotted for completion of the exercise. A score of 1 was given for each correct answer.

Testing sites. On the basis of their schedules and logistic availability, participants were tested in one of two testing environments: 1) an experimental group in which the testing instrument was administered in a natural environment or 2) a control group in which the testing instrument was administered in an artificial environment. The natural environment consisted of a small amphitheater located within a garden area adjacent to a campus building. The garden was composed of a variety of horticultural elements, crushed stone and rock pathways, tiered landscapes, and seating areas including an outdoor teaching theater. The artificial environment was any one of several indoor classrooms. Classrooms were first scrutinized to ensure no elements of a natural environment were present, such as indoor plants or window views of vegetation or landscapes. Participants in the control group met in the scheduled classroom. Participants in the experimental group met in a lobby of the Agriculture Building before being escorted to the outdoor garden.

Test administration. Students in both environments were first asked to review and sign an informed consent document in accordance with the university's institutional review board, and a demographic questionnaire for later use to obtain comparative data. Demographic information collected included the participant's age, gender identity, race/ethnicity, educational level, and degree program. This document was also designed for use as an answer sheet for the subsequent exercises. After completion of the demographic questionnaire, the testing instrument was immediately administered to both groups. To ensure consistency, all tests were administered and scored by the researcher. Tests were administered to students in the outdoor garden during reasonable weather conditions including those days that had no rain, little wind, and temperatures that ranged from 72 to $84^{\circ} \mathrm{F}\left(22.2\right.$ to $\left.28.9^{\circ} \mathrm{C}\right)$. Interior room temperatures where testing occurred were set at 73 to $75{ }^{\circ} \mathrm{F}$ (22.8 to $\left.23.9^{\circ} \mathrm{C}\right)$.

Data Analysis. Scores from the treatment and control groups were compared using the statistical software (SPSS Statistics version 22.0; IBM Corp., Armonk, NY). Statistical analysis included descriptive statistics, frequencies, paired $t$ tests, and analysis of variance tests. 


\section{Results}

SAMPLE. Participants in this study were degree-seeking students of all classifications from diverse academic disciplines at Texas State University, ranging in age from 18 to more than 40 years. The sample came from a variety of majors. The total sample size was 380, of which 190 subjects were tested in the artificial environthe natural environment. A complete description of the sample's demographics can be found in Table 1 .

Treatment vs. CONTROL GROUP COMPARISONS. Exercise 1, a modified form of the SRT, was one of two tests used to evaluate memory. The mean score of the SRT of subjects tested in the artificial environment was 91.77. The mean score of the SRT of subjects tested in the natural environment was 92.73. No significant difference was found to exist between the scores of the two groups $(P=0.399)$.

Exercise 2, a modified form of the CVLT was the second test used to evaluate memory. The mean score of the CVLT of subjects tested in the artificial environment was 25.61. The mean score of the CVLT of subjects tested in the natural environment was ment and 190 subjects were tested in

24.96. No significant difference was found to exist between the scores of the two groups $(P=0.708)$.

An aggregate score was calculated of the two tests used to evaluate memory for each participant. The mean score of the memory totals of subjects tested in the artificial environment was 117.14. The mean score of the memory totals of subjects tested in the natural environment was 117.89. No significant difference was found to exist between the scores of the two groups $(P=0.588)$.

Exercise 3, a modified form of the WAIS-IV Matrix Reasoning Test, was used to evaluate reasoning. The mean score of the WAIS-IV Matrix Reasoning Test of subjects tested in the artificial environment was 9.63. The mean score of the WAIS-IV Matrix Reasoning Test of subjects tested in the natural environment was 9.37. No significant difference was found to exist between the scores of the two groups $(P=0.095)$.

DEMOGRAPHIC COMPARISONS. Demographic categories were compared with investigate whether significant differences existed between the memory and/or reasoning scores of subjects tested in the natural environment and subjects tested in the

Table 1. Breakdown of the sample demographics in the study of the effects of environment on memory and reasoning skills in natural or artificial environments.

\begin{tabular}{lrc}
\hline & \multicolumn{2}{c}{ Study participants } \\
\cline { 2 - 3 } Group & (no.) & $(\%)$ \\
\hline Classification & 213 & \\
$\quad$ Freshman & 49 & 56.0 \\
Sophomore & 54 & 12.8 \\
Junior & 59 & 14.5 \\
Senior & 5 & 15.5 \\
$\quad$ Postgraduate & & 0.01 \\
Ethnicity & 202 & \\
White & 104 & 53.2 \\
Hispanic & 44 & 27.4 \\
Black/African American & 30 & 11.6 \\
Other & & 7.8 \\
Age (years) & 270 & \\
18-20 & 79 & 71.5 \\
21-25 & 21 & 20.7 \\
26-30 & 3 & 5.5 \\
31-35 & 4 & 0.08 \\
36-40 & 3 & 1.0 \\
Over 40 & & 0.08 \\
Gender & 263 & 69.2 \\
Female & 115 & 30.3 \\
Male & 2 & 0.05 \\
Transgender/other & &
\end{tabular}

artificial environment. The analysis of variance comparing those with a grade classification of senior produced a significant probability value $(0.035)$ for the SRT. A comparison of the means between seniors who were tested in a natural environment and those tested in an artificial environment indicated that seniors who were tested in the natural environment performed significantly better on this test compared with other grade levels (Table 2).

\section{Discussion}

The attention restoration theory suggests that directed attention is subject to fatigue and that the presence of nature and natural environments allows recovery from that fatigue, consequently improving cognitive function. In the context of feeling less stressed and less pressured and experiencing a greater degree of restorativeness, Kaplan (1993) indicated that even a glimpse of nature for brief moments can improve those conditions. Regarding cognitive function, a specific amount of time needed to recover from directed-attention fatigue is not addressed, yet the theory inherently necessitates a time element of some duration to allow recovery to occur. Several studies reviewed relating to cognitive function included a period of time in which the subject was exposed to a restorative environment with the intent to induce recovery from directed-attention fatigue or in which the participants had been exposed to the particular environments for long periods of time (Berman et al., 2008; Cimprich, 1993; Hartig et al., 1991). The experimental design of this study intentionally excluded time spent in the presence of nature or a natural environment before testing subjects in the natural setting. Excluding this type of treatment protocol allowed the study to investigate whether memory and reasoning were enhanced when exercised concurrently in the presence of soft fascinations.

Test-taking requires directed attention toward that which one is consciously engaged as well as the suppression of potential distractions. In the natural testing environment, directed attention to the demands of the testing instrument may have suppressed the surrounding soft fascinations, prohibiting their potential 
Table 2. Analysis of variance comparisons of Sentence Repetition Test (Spreen and Benton, 1977) scores of students classified as seniors tested in a natural environment and of students classified as seniors tested in an artificial environment.

\begin{tabular}{lcccccc}
\hline Group & Students (no.) & Mean score $^{\mathrm{z}}$ & SD & df & $\boldsymbol{F}$ & $\boldsymbol{P}$ \\
\hline Natural & 37 & 96.22 & 9.03 & 1 & 4.64 & $0.035^{*}$ \\
Artificial & 22 & 90.45 & 11.31 & & & \\
\hline
\end{tabular}

${ }^{\mathrm{z}}$ Scores range from 0 to 107 , with greater scores indicating a higher degree of sentence recall.

*Statistically significant at $P \leq 0.05$.

restorative effects. The suppression of soft fascinations, and consequently their restorative effects, may account for the findings of no significant differences of the cognitive functions of memory and reasoning between the two groups.

The limitation of time may have influenced the results of the study. Because many of the students were tested during a class period, the time allotted to complete the testing instrument required an accommodation to the shortest class period, which was 50 min. To be consistent, all other subjects were tested within the same time frame. The duration of time to complete the test may not have been sufficient to initiate directed-attention fatigue. A testing instrument of longer duration might initiate fatigue that concurrently could be arrested or suppressed by the surrounding soft fascinations. The difficulty of the testing instrument, as well, might not have been sufficient to initiate fatigue. These potentialities may account for the absence of significant differences within the study.

The limitation of time also prohibited more than one meeting with the subjects, which may have affected the cognitive condition of those tested in the natural environment. In the course of collecting data, an observation was made that subjects tested in the artificial environment appeared comfortable, relaxed, and able to immediately focus on the testing instrument. However, a number of subjects tested in the natural environment appeared stressed, uncomfortable, frustrated, and unable to immediately focus. Many of these subjects arrived late, stating that they had difficulty finding the testing site.

Eighty of the 96 students tested in the natural environment were firstsemester freshmen. A three-question survey was emailed to these 80 freshmen to evaluate their pretest cognitive condition. The first asked whether they had been to the testing site before the testing date. If they answered no, they were asked two subsequent questions. Using a 5-point Likert scale, the first question asked them to rate their level of agreement or disagreement with the statement, "I felt stressed upon arrival as a result of looking for the test site." Using a 5point Likert scale the second question asked them to rate their level of agreement or disagreement with the statement, "I feel my stress level affected my performance on the test." There were 45 responses, 13 reporting they had been to the testing site before the testing date, and 32 reporting they had not. Of these, 16 reported that they agreed or strongly agreed they felt stressed upon arrival as a result of looking for the test site. Fifteen reported that they agreed or strongly agreed their performance on the test was influenced by looking for the test site. Locating the testing site required directed attention. These subjects may have arrived already experiencing a level of directed-attention fatigue. Students tested in a familiar classroom did not experience this fatigue. The failure of the research design to take this factor into consideration may have created a confounding variable that influenced the findings and may account for the absence of significant differences between most groups.

The participants who performed significantly better on the SRT were those with a grade classification of senior. This cohort represented individuals who may have developed more efficient listening skills, memorization strategies, stress management, test-taking techniques, and greater self-confidence because of their collegiate academic experiences. These enhanced abilities may have reduced the effort expended for both directed attention and the suppression of distractions while performing the task allowing the soft fascinations to be concurrently experienced and a restorative effect realized.

\section{Applications}

Effectual cognitive function is essential for success in education, business, and our personal lives, and the attention restoration theory presents practical strategies to improve cognitive function. Programming of interior and exterior space during the construction or renovation of educational facilities should focus on affording the greatest degree of exposure to nature and the natural environment as possible. Landscaped pedestrian pathways and parks, outdoor teaching venues and common areas, window views of green space or interior designs including plants, nature paintings, and murals may be effective strategies for students, faculty, and staff to reduce and/or recover from directedattention fatigue.

Faculty should consider incorporating recovery time in their teaching protocols. Kaplan (1993) suggested that the opportunity to move one's focus away from work and experience nature, even for brief moments, can help recovery from directed-attention fatigue. Recovery time might include short breaks scheduled throughout the lecture or laboratory with students being encouraged to momentarily redirect their focus. Ideally, this focus would be directed toward the surrounding areas programmed with elements of nature.

Additionally, personal space, office buildings, retail spaces, and even industrial facilities should model this paradigm of exposure to nature and natural environments. Enhancing cognitive function and reducing directed-attention fatigue may improve decisionmaking, efficiency, productivity, and even customer service. A comprehensive list of strategies is beyond the scope of this work, but one can begin to appreciate the potential benefits that can be realized.

\section{Conclusions}

The research design of this study may have created a confounding variable that influenced the findings. Some subjects may have arrived at the testing site experiencing some degree of directed-attention fatigue, whereas others did not. Strategies to ensure consistent baselines among subjects should be considered. In this case, the limitation of time prevented meeting the subjects being tested in the natural 
environment before the testing date. Having first met at the testing site before the testing date would have allowed subjects to familiarize and orient themselves to the location and may have prevented this phenomenon.

Although this study returned mixed results, research generally supports the premise that exposure to nature or natural environments can have beneficial effects on physical and mental health as well as improve cognitive function. Further studies should possibly include more than one meeting time to alleviate the potential confounding variable presented in this study, time for participants to sit and observe in the natural vs. artificial environment before testing, and increased sample sizes that would allow further demographic comparisons. Additionally, students may have existing stress and/or anxiety, which may influence testing and results, which should be included as a factor in future studies.

Effectual cognitive function is essential for success in education, business, and our personal lives. Although the attention restoration theory presents practical strategies to recover from directed-attention fatigue and improve cognitive function, better understanding of the mechanisms of directed-attention fatigue and restoration, the impact of directed-attention fatigue on cognitive function, and the most efficient ways to measure them are needed.

\section{Literature cited}

Adamson, K. and A. Thatcher. 2018. Do indoor plants improve performance outcomes? Using the attention restoration theory. Cong. Internatl. Ergon. Assoc. 591-604, doi: 1007/978-3-319-96068-5-65.

Berman, M.G., J. Jonides, and S. Kaplan. 2008. The cognitive benefits of interacting with nature. Psychol. Sci. 19(12):12071212, doi: $10.111 /$ j.1467-9280.2008.02 225.x.

Bleeker, M.L., K. Bolla-Wilson, J. Agnew, and D. Meyers. 1988. Age-related sex differences in verbal memory. Neuropsychol. Assess. 44(3):403-411, doi: 10.1002/1097-4679(199805)44:3.

Cimprich, B. 1993. Development of an intervention to restore attention in cancer patients. Cancer Nurs. 16(2):83-92, doi: 10/1097/00002820-199304000-00001.

Conway, A.R., M.J. Kane, and R.W. Engle. 2003. Working memory capacity and its relation to general intelligence. Trends Cogn. Sci. 7(12):547-552, doi: 10.1016/j.tics.2003.10.05.

Crossan, C. and A. Salmoni. 2021. A simulated walk in nature: Testing predictions from the attention restoration theory. Environ. Behav. 53(3):277-295, doi: $10.1177 / 0013916519882775$.

Delis, D.C. 2000. California verbal learning test. 2nd ed. Pearson, Bloomington, $\mathrm{MN}$.

Felsten, G. 2009. Where to take a study break on the college campus: An attention restoration theory perspective. J. Environ. Psychol. 29(1):160-167, doi: 10.1016/j. envp.2008.11.006.

Hartig, T., M. Mang, and G.W. Evans. 1991. Restorative effects of natural environment experiences. Environ. Behav. 23(1):3-26, doi: 10.1177/0013916591 231001.

Hartig, T., K. Korpela, G.W. Evans, and T. Gärling. 1997. A measure of restorative quality in environments. Scand. Hous. Plan. Res. 14(4):175-194, doi: 10.1080/ 02815739708730435.

Herzog, T.R., A.M. Black, K.A. Fountaine, and D.J. Knotts. 1997. Reflection and attentional recovery as distinctive benefits of restorative environments. J. Environ. Psychol. 17(2):165-170, doi: 10.1006/jevp.1997.0051.

Jaeggi, S.M., M. Buschkeul, J. Jonides, and W.J. Perrig. 2008. Improving fluid intelligence with training on working memory. Proc. Natl. Acad. Sci. USA 105(19):68296833, doi: 10:1073/pnas.081268105.

James, W. 1892. Psychology: The briefer course. Henry Holt, New York, NY.

Jiang, B., J. He, J. Chen, L. Larsen, and H. Wang. 2021. Perceived green at speed: A simulated driving experiment raises new questions for attention restoration theory and stress reduction theory. Environ. Behav. 53(3):296-335, doi: 10.1177/ 0013916520947111.

Kaplan, R. 1993. The role of nature in the context of the workplace. Lands. Urb. Plan. 26(1-4):193-201, doi: 10:1016/ 0169-2046(93)90016-7.

Kaplan, R. and S. Kaplan. 1989. The experience of nature: A psychological perspective. Cambridge Univ. Press, New York, NY.

Kaplan, S. 1995. The restorative benefits of nature: Toward an integrative framework. J. Environ. Psychol. 15(3):169-182, doi: 10/1016/0272-0272-4944(95)90001-2.

Krejcie, R.V. and D.W. Morgan. 1970. Determining sample size for research activities. Educ. Psychol. Meas. 30(3):607-610, doi: 10/1177/001316447003000308.

Laumann, K., T. Gärling, and K.M. Stormark. 2001. Rating scale measures of restorative components of environments. J. Environ. Psychol. 21(1):31-44, doi: 10.1006/jevp.20000179.

Moreno, A., S. Baker, K. Varey, and R. Hinze-Pifer. 2018. Bringing attention restoration theory to the classroom: A tablet app using nature videos to replenish effortful cognition. Trends Neurosci. Educ. 12:7-21, doi: 10.1016/j.tinc.2018.07.002.

Purcell, T., E. Peron, and R. Berto. 2001. Why do preferences differ between scene types? Environ. Behav. 33(1):93-106, doi: 10.1077/00139160121972882.

Shin, W.S., C.S. Shin, P.S. Yeoun, and J.J. Kim. 2011. The influence of interaction with forest on cognitive function. Scand. J. For. Res. 26(6):595-598, doi: $10.1080 / 02827581.2011 .585996$.

Spreen, O. and A. Benton. 1977. Neurosensory center comprehensive examination for aphasia. Victoria Neuropsychol. Lab., Victoria, BC, Canada.

Tennessen, C.M. and B. Cimprich. 1995. Views to nature: Effects on attention. J. Environ. Psychol. 15(1):77-85, doi: 10.1016/0272-4944(95)90016-0.

Wechsler, D. 2014. Wechsler adult intelligence scale-fourth edition (WAIS-IV). Pearson, Bloomington, $\mathrm{MN}$. 